\title{
Finite polytopes have finite regular covers
}

\author{
B. Monson · Egon Schulte
}

Received: 20 September 2012 / Accepted: 12 September 2013 / Published online: 5 October 2013

(C) Springer Science+Business Media New York 2013

\begin{abstract}
We prove that any finite, abstract $n$-polytope is covered by a finite, abstract regular $n$-polytope.
\end{abstract}

Keywords Abstract polytopes $\cdot$ Regular polytopes $\cdot$ Covers $\cdot$ Monodromy group

\section{Introduction}

Any point or line segment is a regular convex polytope, admittedly of modest dimension. And every polygon with $p$ vertices is combinatorially equivalent to a regular $p$-gon. Moving further into the combinatorial domain, it is clear that a typical map $\mathcal{M}$ on a compact surface is nonregular. Even so, it is folklore (and not so hard to prove, see [5, Corollary 6.2]) that $\mathcal{M}$ is a quotient of a regular map $\mathcal{R}$ of finite type $\{p, q\}$, most likely on some new surface, and which, from another point of view, covers the base map $\mathcal{M}$.

On the other hand, it is certainly true that any $n$-polytope is a quotient of the universal regular $n$-polytope $\mathcal{U}_{n}=\{\infty, \ldots, \infty\}$, which is indeed infinite if the rank $n \geq 2$.

In this note we look at all these situations from an abstract point of view and prove a reasonable but hitherto unaddressed result: every finite abstract $n$-polytope $\mathcal{Q}$

B. Monson was supported in part by PAPIIT-Mexico grant \# IN112512 and by NSERC of Canada Discovery Grant \# 4818.

E. Schulte was supported by NSF-grant DMS-0856675.

B. Monson

University of New Brunswick, Fredericton, NB E3B 5A3, Canada

E. Schulte $(\bowtie)$

Northeastern University, Boston, MA 02115, USA

e-mail: schulte@neu.edu 
has a finite regular cover $\mathcal{R}$ (Theorem 3.4). This particular finite cover problem has been raised by one of us (B.M.) at various conferences (see also [7] for some related questions). In essence Theorem 3.4 says that there exists a covering map from the infinite polytope $\mathcal{U}_{n}$ onto $\mathcal{Q}$ which factors through a finite regular $n$-polytope. Thus the covering relationship between $\mathcal{U}_{n}$ and $\mathcal{Q}$ can be described as a sequence of two covering relationships: the first between two regular polytopes, namely $\mathcal{U}_{n}$ and $\mathcal{R}$, and the second between two finite polytopes, namely $\mathcal{R}$ and $\mathcal{Q}$.

\section{Abstract polytopes and their automorphism groups}

An abstract $n$-polytope $\mathcal{P}$ has certain key combinatorial properties of the face lattice of a convex $n$-polytope; in general, however, $\mathcal{P}$ need not be a lattice or have a familiar geometric realization. Let us summarize some general definitions and results, referring to [4] for details. An abstract n-polytope $\mathcal{P}$ is a partially ordered set with properties $\mathbf{A}, \mathbf{B}$ and $\mathbf{C}$ below.

A: $\mathcal{P}$ has a strictly monotone rank function with range $\{-1,0, \ldots, n\}$. Moreover, $\mathcal{P}$ has a unique least face $F_{-1}$ and unique greatest face $F_{n}$.

An element $F \in \mathcal{P}$ with $\operatorname{rank}(F)=j$ is called a $j$-face; often $F_{j}$ will indicate a $j$-face. Each maximal chain or flag in $\mathcal{P}$ therefore contains $n+2$ faces, so that $n$ is the number of proper faces in each flag. We let $\mathcal{F}(\mathcal{P})$ be the set of all flags in $\mathcal{P}$. Naturally, faces of ranks 0,1 and $n-1$ are called vertices, edges and facets, respectively.

B: Whenever $F<G$ with $\operatorname{rank}(F)=j-1$ and $\operatorname{rank}(G)=j+1$, there are exactly two $j$-faces $H$ with $F<H<G$.

For $0 \leq j \leq n-1$ and any flag $\Phi$, there thus exists a unique adjacent flag $\Phi^{j}$, differing from $\Phi$ in just the face of rank $j$. With this notion of adjacency, $\mathcal{F}(\mathcal{P})$ becomes the vertex set of the flag graph for $\mathcal{P}$. If $F \leq G$ are incident faces in $\mathcal{P}$, we call

$$
G / F:=\{H \in \mathcal{P} \mid F \leq H \leq G\}
$$

a section of $\mathcal{P}$.

C: $\mathcal{P}$ is strongly flag-connected, that is, the flag graph for each section is connected.

It follows that $G / F$ is a $(k-j-1)$-polytope in its own right if $F \leq G$ with $\operatorname{rank}(F)=j \leq k=\operatorname{rank}(G)$. For example, if $F$ is a vertex, then the section $F_{n} / F$ is called the vertex-figure over $F$. Likewise, it is useful to think of the $k$-face $G$ as having the structure of the $k$-polytope $G / F_{-1}$.

A map $\varphi: \mathcal{P} \rightarrow \mathcal{Q}$ between $\mathcal{P}$ and another polytope $\mathcal{Q}$ is called a homomorphism if $\varphi$ preserves incidence in one direction; that is, $F \leq G$ in $\mathcal{P}$ implies $F \varphi \leq G \varphi$ in $\mathcal{Q}$. An automorphism of $\mathcal{P}$ is a bijective homomorphism of $\mathcal{P}$ onto itself whose inverse is also a homomorphism.

The automorphism group $\Gamma(\mathcal{P})$ consists of all order-preserving bijections on $\mathcal{P}$. We say $\mathcal{P}$ is regular if $\Gamma(\mathcal{P})$ is transitive on the flag set $\mathcal{F}(\mathcal{P})$. In this case we may 
choose any one flag $\Phi \in \mathcal{F}(\mathcal{P})$ as base flag, then define $\rho_{j}$ to be the (unique) automorphism mapping $\Phi$ to $\Phi^{j}$, for $0 \leq j \leq n-1$. Each $\rho_{j}$ has period 2. From [4, 2B] we recall that $\Gamma(\mathcal{P})$ is then a string $C$-group, meaning that it has the following properties SC1 and SC2:

SC1: $\Gamma(\mathcal{P})$ is generated by $\left\{\rho_{0}, \ldots, \rho_{n-1}\right\}$. These involutory generators satisfy the commutativity relations typical of a Coxeter group with string diagram, namely

$$
\left(\rho_{j} \rho_{k}\right)^{p_{j k}}=1 \text { for } 0 \leq j \leq k \leq n-1 \text {, }
$$

where $p_{j j}=1$ and $p_{j k}=2$ whenever $|j-k|>1$. In other words, $\Gamma(\mathcal{P})$ is a string group generated by involutions or an sggi.

SC2: $\Gamma(\mathcal{P})$ satisfies the intersection condition

$$
\begin{aligned}
& \left\langle\rho_{k}: k \in I\right\rangle \cap\left\langle\rho_{k}: k \in J\right\rangle=\left\langle\rho_{k}: k \in I \cap J\right\rangle \\
& \quad \text { for any } I, J \subseteq\{0, \ldots, n-1\} .
\end{aligned}
$$

The fact that one can reconstruct a regular polytope in a canonical way from any string C-group $\Gamma$ is at the heart of the theory [4,2E]. Thus a regular polytope can be identified naturally with a string $\mathrm{C}$-group. We frequently make use of this fact, and depending on context, work with string $\mathrm{C}$-groups rather than polytopes.

The periods $p_{j}:=p_{j-1, j}$ in (1) satisfy $2 \leq p_{j} \leq \infty$ and are assembled into the Schläfli symbol $\left\{p_{1}, \ldots, p_{n-1}\right\}$ for the regular polytope $\mathcal{P}$. We also say that $\mathcal{P}$ has type indicated by the same symbol and that the group $\Gamma(\mathcal{P})$ has rank $n$.

In the same way, any sggi $G=\left\langle g_{0}, \ldots, g_{n-1}\right\rangle$ also has a Schläfli symbol, type and rank, although $p_{j}=1$ can occur (if $g_{j-1}=g_{j}$; this is impossible for regular polytopes by (2)).

The dual $\mathcal{P}^{*}$ of the polytope $\mathcal{P}$ is obtained by simply reversing the partial order on the underlying set of faces. If $\mathcal{P}$ is regular of type $\left\{p_{1}, \ldots, p_{n-1}\right\}$, then $\mathcal{P}^{*}$ is also regular of type $\left\{p_{n-1}, \ldots, p_{1}\right\}$.

Definition 2.1 [4, 2D] Let $\mathcal{R}$ and $\mathcal{P}$ be $n$-polytopes. A covering is a rank and adjacency preserving homomorphism $\eta: \mathcal{R} \rightarrow \mathcal{P}$. (This means that $\eta$ induces a mapping $\mathcal{F}(\mathcal{R}) \rightarrow \mathcal{F}(\mathcal{P})$ that sends any $j$-adjacent pair of flags in $\mathcal{R}$ to another such pair in $\mathcal{P} ;$ it is easy to show that $\eta$ must then be surjective.) We also say that $\mathcal{R}$ is a cover of $\mathcal{P}$ and write $\mathcal{R} \rightarrow \mathcal{P}$.

In verifying that a given sggi (a group satisfying (1)) is a string C-group, the following quotient criterion is frequently useful (see [4, Thm. 2E17]).

Lemma 2.2 Let $\Gamma=\left\langle\rho_{0}, \ldots, \rho_{n-1}\right\rangle$ be an sggi, and let $\Delta=\left\langle\sigma_{0}, \ldots, \sigma_{n-1}\right\rangle$ be a string $C$-group (with respect to the distinguished generators $\sigma_{i}$ ). If the mapping $\rho_{j} \rightarrow$ $\sigma_{j}$ for $j=0, \ldots, n-1$ induces a homomorphism $\pi: \Gamma \rightarrow \Delta$, which is one-to-one on $\left\langle\rho_{0}, \ldots, \rho_{n-2}\right\rangle$ or on $\left\langle\rho_{1}, \ldots, \rho_{n-1}\right\rangle$, then $\Gamma$ is also a string $C$-group, and $\pi$ induces $a$ covering $\mathcal{R} \rightarrow \mathcal{P}$ between the regular polytopes $\mathcal{R}$ and $\mathcal{P}$ corresponding to $\Gamma$ and $\Delta$, respectively.

If a polytope $\mathcal{R}$ covers a polytope $\mathcal{P}$, then from another point of view, $\mathcal{P}$ will be a quotient of $\mathcal{R}$; see [4, 2D], [3] or [5]. One way to understand how $\mathcal{P}$ arises by identifications in $\mathcal{R}$ is to exploit the monodromy group: 
Definition 2.3 Let $\mathcal{P}$ be a polytope of rank $n \geq 1$. For $0 \leq j \leq n-1$, let $g_{j}$ be the bijection on $\mathcal{F}(\mathcal{P})$ that maps each flag $\Phi$ to the $j$-adjacent flag $\Phi^{j}$. Then the monodromy group for $\mathcal{P}$ is

$$
\operatorname{Mon}(\mathcal{P})=\left\langle g_{0}, \ldots, g_{n-1}\right\rangle
$$

(a subgroup of the symmetric group on $\mathcal{F}(\mathcal{P})$ ).

It is easy to see that $\operatorname{Mon}(\mathcal{P})$ is an sggi. Let us quote from [5] some useful and fairly easily proved results. We let $\operatorname{Stab}_{\operatorname{Mon}(\mathcal{P})} \Phi$ denote the stabilizer of a flag $\Phi$ under the action of $\operatorname{Mon}(\mathcal{P})$.

Theorem 2.4 Let $\mathcal{R}$ be a regular $n$-polytope with base flag $\Phi$, automorphism group $\Gamma(\mathcal{R})=\left\langle\rho_{0}, \ldots, \rho_{n-1}\right\rangle$, and monodromy group $\operatorname{Mon}(\mathcal{R})=\left\langle g_{0}, \ldots, g_{n-1}\right\rangle$. Then there is an isomorphism $\Gamma(\mathcal{R}) \simeq \operatorname{Mon}(\mathcal{R})$ mapping each $\rho_{j}$ to $g_{j}$.

Theorem 2.5 Suppose that $\mathcal{R}$ and $\mathcal{Q}$ are n-polytopes and that

$$
\bar{\eta}: \operatorname{Mon}(\mathcal{R}) \rightarrow \operatorname{Mon}(\mathcal{Q})
$$

is an epimorphism of sggi's (i.e. mapping specified generators to specified generators, in order). Suppose also that there are flags $\Lambda^{\prime}$ of $\mathcal{R}$ and $\Lambda$ of $\mathcal{Q}$ such that

$$
\left(\operatorname{Stab}_{\operatorname{Mon}(\mathcal{R})} \Lambda^{\prime}\right) \bar{\eta} \subseteq \operatorname{Stab}_{\operatorname{Mon}(\mathcal{Q})} \Lambda .
$$

Then there is a unique covering $\eta: \mathcal{R} \rightarrow \mathcal{Q}$ that maps $\Lambda^{\prime}$ to $\Lambda$.

Remark 2.6 If $\mathcal{R}$ is regular, then condition (3) is fulfilled automatically, since all flags $\Lambda^{\prime}$ are equivalent, with trivial stabilizer, in $\Gamma(\mathcal{R}) \simeq \operatorname{Mon}(\mathcal{R})$ (Theorem 2.4).

\section{Regular covers of general polytopes}

A key step in our construction is provided by extension results for regular polytopes. By an extension of an $m$-polytope $\mathcal{K}$ we mean an $(m+1)$-polytope all of whose facets are isomorphic to $\mathcal{K}$. There are many ways of extending a polytope.

First there is the trivial way. Any $m$-polytope $\mathcal{K}$ is the facet type of an $(m+1)$ polytope with only two facets, here denoted $\{\mathcal{K}, 2\}$ (for example, see [4, 8]). If $\mathcal{K}$ is regular, then $\{\mathcal{K}, 2\}$ is also regular, and $\Gamma(\{K, 2\})=\Gamma(\mathcal{K}) \times C_{2}$, where the $C_{2}$-factor is generated by the $(m+1)$-st distinguished generator.

The construction of extensions becomes more complicated if further conditions are imposed on the new polytope. The following lemma and its proof are a variation of Danzer's $2^{K}$ construction (see [4, Ch. 8C]). Even though later we mainly require the trivial extension of $\mathcal{K}$ just described (rather than that of Lemma 3.1), we will reproduce here some of the details of the $2^{\mathcal{K}}$ construction in a form that is slightly different from presentations that have appeared elsewhere.

Lemma 3.1 Suppose $\mathcal{K}$ is a regular $m$-polytope with automorphism group $\Gamma(\mathcal{K})=$ $\left\langle\rho_{0}, \ldots, \rho_{m-1}\right\rangle$, Schläfli type $\left\{p_{1}, \ldots, p_{m-1}\right\}$ and facet set $A$. Then there is a regular 
$(m+1)$-polytope $\overline{\mathcal{K}}$ of type $\left\{p_{1}, \ldots, p_{m-1}, 4\right\}$, with facets isomorphic to $\mathcal{K}$, and such that $|\Gamma(\overline{\mathcal{K}})|=|\Gamma(\mathcal{K})| \cdot 2^{|A|}$, when $\mathcal{K}$ is finite. Indeed, $\overline{\mathcal{K}}$ is finite if and only if $\mathcal{K}$ is finite.

Proof As hinted above, we could simply refer to [4, Theorem 8C2] and take $\overline{\mathcal{K}}=\left(2^{\mathcal{K}^{*}}\right)^{*}$. But for the reader's convenience, we shall rework here the essentials of that construction. First off, let $N$ be the group of all sequences

$$
x: A \rightarrow C_{2}:=\{ \pm 1\},
$$

with component-wise multiplication. (If $A$ is infinite, take sequences of finite "support", meaning that -1 can occur only finitely often.) Thus $N$ is generated by the indicator functions $\delta_{F}, F \in A$, where for facets $F, H \in A$, we have

$$
(H) \delta_{F}= \begin{cases}-1 & \text { if } H=F, \\ +1 & \text { if } H \neq F .\end{cases}
$$

We require $\rho_{m}:=\delta_{F_{m-1}}$, the indicator function for the base facet of $\mathcal{K}$.

Now for $x \in N, \gamma \in \Gamma(\mathcal{K}), F \in A$, we let

$$
(F) x^{\gamma}:=(F) \gamma^{-1} x .
$$

This defines an action $x \mapsto x^{\gamma}$ of $\Gamma(\mathcal{K})$ on $N$, and we let

$$
S:=N \rtimes \Gamma(\mathcal{K})
$$

be the corresponding semidirect (indeed, wreath) product. For ready computation, we abuse notation a bit, taking $N \triangleleft S$ and $\Gamma(\mathcal{K})<S$, so that $x \cdot \gamma=\gamma \cdot x^{\gamma}$ for $x \in N, \gamma \in \Gamma(\mathcal{K})$. Note in particular that $\delta_{F}^{\gamma}=\delta_{(F) \gamma}$ for any facet $F$. Since the base facet $F_{m-1}$ is stabilized by $\rho_{j}, 0 \leq j \leq m-2$, we have

$$
\rho_{j} \rho_{m}=\rho_{j} \delta_{F_{m-1}}=\delta_{F_{m-1}}^{\rho_{j}} \rho_{j}=\delta_{\left(F_{m-1}\right) \rho_{j}} \rho_{j}=\delta_{\left(F_{m-1}\right)} \rho_{j}=\rho_{m} \rho_{j} .
$$

Similarly we find that $\left(\rho_{m-1} \rho_{m}\right)^{2}$ is an element of $N$ and so has period 2. In fact,

$$
S=\left\langle\rho_{0}, \ldots, \rho_{m-1}, \rho_{m}\right\rangle
$$

is an sggi of rank $m+1$ and type $\left\{p_{1}, \ldots, p_{m-1}, 4\right\}$. Using the unique factorization in $S$ given by $S=N \Gamma(\mathcal{K})$, together with the intersection property (2) for $\mathcal{K}$, we soon verify the intersection property for $S$, too [4, Lemma 8B5]. Thus $S$ is a string C-group of rank $m+1$, so $S=\Gamma(\overline{\mathcal{K}})$ for just the sort of regular $(m+1)$-polytope $\overline{\mathcal{K}}$ which we seek.

Remark 3.2 In [6], Daniel Pellicer uses 'CPR-graphs' to generalize the results in Lemma 3.1. One can construct a regular polytope $\overline{\mathcal{K}}$ of type $\left\{p_{1}, \ldots, p_{m-1}, 2 s\right\}$, with facets isomorphic to $\mathcal{K}$, for any integer $s \geq 2$.

Lemma 3.3 Let $G=\left\langle g_{0}, \ldots, g_{n-1}\right\rangle$ be an sggi, and let $0 \leq i \leq n-2$. Suppose there exists a string $C$-group $H=\left\langle h_{0}, \ldots, h_{i}\right\rangle$ that covers $\left\langle g_{0}, \ldots, g_{i}\right\rangle$. Then there also exists a string $C$-group $L=\left\langle l_{0}, \ldots, l_{i+1}\right\rangle$ that covers $\left\langle g_{0}, \ldots, g_{i+1}\right\rangle$. Furthermore, we may choose $L$ in such a way that, if $j \leq i$ and $\left\langle g_{0}, \ldots, g_{j}\right\rangle \simeq\left\langle h_{0}, \ldots, h_{j}\right\rangle$, then $\left\langle g_{0}, \ldots, g_{j}\right\rangle \simeq\left\langle l_{0}, \ldots, l_{j}\right\rangle$ as well. Moreover, if $\left\langle g_{0}, \ldots, g_{i+1}\right\rangle$ and $H$ are finite, then we can take $L$ to be finite, too. 
Proof Let $\mathcal{K}$ be a regular $(i+1)$-polytope with $\Gamma(\mathcal{K}) \simeq H$. Then there is a regular $(i+2)$-polytope $\overline{\mathcal{K}}$ with facets isomorphic to $\mathcal{K}$. In fact, we can choose any such regular $(i+2)$-polytope for $\overline{\mathcal{K}}$; for example, the trivial extension $\{K, 2\}$ described earlier, ${ }^{1}$ or the polytope of Lemma 3.1. Either way, we may suppose that $\Gamma(\overline{\mathcal{K}})=$ $\left\langle h_{0}, \ldots, h_{i}, h_{i+1}\right\rangle$.

Now we exploit the fact that the mix of two sggi's is the smallest sggi that covers both sggi's. More explicitly, the mix $L=\left\langle g_{0}, \ldots, g_{i+1}\right\rangle \diamond\left\langle h_{0}, \ldots, h_{i+1}\right\rangle$ (of $\left\langle g_{0}, \ldots, g_{i+1}\right\rangle$ and $\left\langle h_{0}, \ldots, h_{i+1}\right\rangle$ ) is the subgroup of the direct product

$$
\left\langle g_{0}, \ldots, g_{i+1}\right\rangle \times\left\langle h_{0}, \ldots, h_{i+1}\right\rangle=\left\langle g_{0}, \ldots, g_{i+1}\right\rangle \times \Gamma(\overline{\mathcal{K}})
$$

generated by all $l_{t}:=\left(g_{t}, h_{t}\right), 0 \leq t \leq i+1$. Clearly, $L$ is also an sggi of rank $i+2$. (We refer to $[2,4,5,10]$ for other useful properties of this operation.)

Since $H$ covers $\left\langle g_{0}, \ldots, g_{i}\right\rangle$, we have $H \simeq\left\langle l_{0}, \ldots, l_{i}\right\rangle$, too. Thus we can apply the quotient criterion of Lemma 2.2 to the second natural projection $L \rightarrow \Gamma(\overline{\mathcal{K}})$ (onto the second component of the mix), and conclude that $L$ is a string C-group. The first natural projection shows that $L$ covers $\left\langle g_{0}, \ldots, g_{i+1}\right\rangle$.

Note that if $j \leq i$ and $f:\left\langle g_{0}, \ldots, g_{j}\right\rangle \rightarrow\left\langle h_{0}, \ldots, h_{j}\right\rangle$ is an isomorphism of sggi's, then the map $g_{t} \mapsto\left(g_{t},\left(g_{t}\right) f\right)=l_{t}, 0 \leq t \leq j$, clearly induces an isomorphism of $\left\langle g_{0}, \ldots, g_{j}\right\rangle$ with $\left\langle l_{0}, \ldots, l_{j}\right\rangle$.

Appealing once more to Lemma 3.1, we see that $L$ is finite if both factors in the direct product (4) are finite.

The last lemma is just what we need to prove our main result.

\section{Theorem 3.4}

(a) Every finite sggi $G$ is covered by a finite string $C$-group $G^{\prime}$.

(b) Every finite n-polytope $\mathcal{Q}$ is covered by a finite regular $n$-polytope $\mathcal{R}$. If $\mathcal{Q}$ has all its $k$-faces isomorphic to some regular $k$-polytope $\mathcal{K}$, then we may choose $\mathcal{R}$ to have its $k$-faces isomorphic to $\mathcal{K}$.

Proof Let $G=\left\langle g_{0}, \ldots, g_{n-1}\right\rangle$ be any finite sggi. Clearly, $H_{0}:=\left\langle g_{0}\right\rangle$ is a string Cgroup, as is the dihedral group $H_{1}:=\left\langle g_{0}, g_{1}\right\rangle$. Thus we can begin an inductive construction. Suppose $H_{j}$ is a finite string C-group covering $\left\langle g_{0}, \ldots, g_{j}\right\rangle$. Take $i=j$ and $H=H_{j}$ in Lemma 3.3. We obtain a finite string C-group $L$ covering $\left\langle g_{0}, \ldots, g_{j+1}\right\rangle$. Now let $H_{j+1}=L$ and iterate. Eventually we get a finite string C-group $G^{\prime}=H_{n-1}$ that covers $G$. Note that if $\left\langle g_{0}, \ldots, g_{k-1}\right\rangle$ happens to be a string C-group, we can start the iteration with this subgroup as our choice for $H$. Then it is clear from Lemma 3.3 that at each stage of the iteration the corresponding subgroup of the mix is isomorphic to $\left\langle g_{0}, \ldots, g_{k-1}\right\rangle$ and that therefore we end with the corresponding subgroup of $G^{\prime}$ isomorphic to $\left\langle g_{0}, \ldots, g_{k-1}\right\rangle$ as well.

For part (b), we merely apply part (a) to the finite $\operatorname{sggi} G=\operatorname{Mon}(\mathcal{Q})=$ $\left\langle g_{0}, \ldots, g_{n-1}\right\rangle$. Let $\mathcal{R}$ be the finite regular $n$-polytope whose automorphism group

\footnotetext{
${ }^{1}$ We thank an anonymous referee for suggesting the use of the trivial extension. This greatly simplifies many explicit calculations.
} 
is $G^{\prime}$ constructed in (a). From Theorem 2.5 we conclude that $\mathcal{R}$ covers $\mathcal{Q}$. If all $k$ faces are isomorphic to $\mathcal{K}$, it is also true that $\left\langle g_{0}, \ldots, g_{k-1}\right\rangle \simeq \Gamma(\mathcal{K})$ [5]. We conclude that $\mathcal{R}$ has isomorphic $k$-faces.

Remark 3.5 It is clear that a dual result concerning co- $k$-faces must hold in Theorem 3.4(b).

Corollary 3.6 Every convex n-polytope $\mathcal{Q}$ has a finite abstract regular cover $\mathcal{R}$. If $\mathcal{Q}$ is simplicial (or simple), then $\mathcal{R}$ is likewise simplicial (or simple).

The monodromy group $G$ of a polytope $\mathcal{Q}$ always gives rise to a regular prepolytopal cover $\mathcal{V}$, constructed from $G$ as a coset geometry in much the same way as a regular polytope can be rebuilt from a given string $\mathrm{C}$-group $\Gamma$. However, as the following example shows, this object $\mathcal{V}$ can fail condition $\mathbf{C}$ concerning strong flag-connectedness.

Example 3.7 Suppose $\mathcal{Q}$ is a pyramid over the toroidal base $\{4,4\}_{(3,0)}$. Recall that $\{4,4\}_{(3,0)}$ is the map on the 2-torus obtained from a $3 \times 3$ chessboard by identifying opposite sides (see [1]). Now the 'lateral' facets of the self-dual 4-polytope $\mathcal{Q}$ are the nine ordinary pyramids over the square faces in the toroid. Using GAP [9], we find that the monodromy group $G=\left\langle g_{0}, g_{1}, g_{2}, g_{3}\right\rangle$ is an sggi of type $\{12,12,12\}$ and order $2^{12} \cdot 3^{11} \cdot 5$. However, the intersection condition (2) fails since $\left\langle g_{1}, g_{2}\right\rangle$ has index 2 in $\left\langle g_{0}, g_{1}, g_{2}\right\rangle \cap\left\langle g_{1}, g_{2}, g_{3}\right\rangle$. Following our earlier remarks, we could manufacture a regular pre-polytopal cover $\mathcal{V}$ of $\mathcal{Q}$ with automorphism group $G$. We find, however, that the section between a typical vertex and facet of $\mathcal{V}$ consists of $t$ wo disjoint copies of a dodecagon $\{12\}$.

If we want a finite, regular polytopal cover $\mathcal{R}$, then we must appeal to Theorem 3.4 and its proof. Since the subgroup $\left\langle g_{0}, g_{1}, g_{2}\right\rangle$ is a string C-group (of order $2^{12} \cdot 3^{3}$ ), we actually need to employ an extension of the corresponding regular 3 -polytope $\mathcal{K}$ just once. This polytope $\mathcal{K}$ has 4608 facets $\{12\}$.

The regular extension $\overline{\mathcal{K}}$ of $\mathcal{K}$ described in Lemma 3.1 has type $\{12,12,4\}$ with group order $|\Gamma(\overline{\mathcal{K}})|=2^{12} \cdot 3^{3} \cdot 2^{4608}$. The resulting regular cover $\mathcal{R}$ still has type $\{12,12,12\}$, with facets isomorphic to $\mathcal{K}$. Note that this particular regular cover of $\mathcal{Q}$ is quite large, with a group order bounded by

$$
|G \times \Gamma(\overline{\mathcal{K}})|=2^{4632} \cdot 3^{14} \cdot 5 .
$$

On the other hand, if $\overline{\mathcal{K}}$ is the trivial regular extension $\{\mathcal{K}, 2\}$ described earlier, then $\overline{\mathcal{K}}$ is of type $\{12,12,2\}$. The corresponding regular cover $\mathcal{R}$ again has type $\{12,12,12\}$, but now its automorphism group is much smaller than in the previous case and has order $2^{23} \cdot 3^{14} \cdot 5$.

It can be shown that there are (at least) two mutually non-isomorphic minimal regular covers of the 4-polytope $\mathcal{Q}$. Each has type $\{12,12,12\}$ and a group of or$\operatorname{der} 2^{13} \cdot 3^{11} \cdot 5$, which is twice the order of the monodromy group of $\mathcal{Q}$. It may be conjectured that these two are the only minimal regular covers of $\mathcal{Q}$.

Remark 3.8 One might ask whether there is some sort of extension of Theorem 3.4(b) to the class of infinite polytopes $\mathcal{Q}$. Perhaps each has a regular cover with finite 'covering index'. 
To see that there is no hope for a general statement of this sort, we begin with $\mathcal{T}=\{4,4\}$. This familiar tiling of the Euclidean plane $\mathbb{R}^{2}$ by unit squares is an infinite regular 3-polytope. Next, for every odd integer $n \geq 3$, we subdivide the square with southwest vertex $(n, 0)$, using an $(n+2)$-gon together with $n$ triangles. The southeast vertex now has degree $n+3$. (The figure illustrates the case $n=5$.) The resulting 3 polytope $\mathcal{Q}$ clearly has a trivial automorphism group $\Gamma(\mathcal{Q})$.

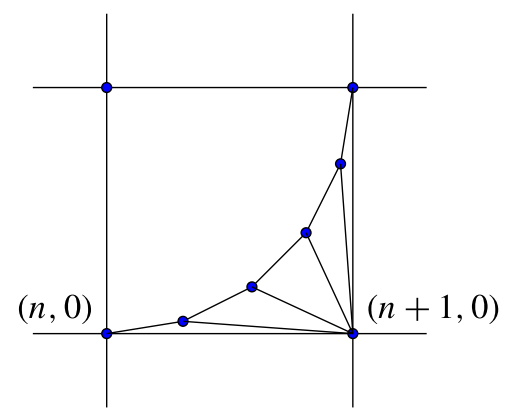

Even though $\mathcal{Q}$ is locally finite, it is also clear that any regular cover $\mathcal{R}$ must have Schläfli type $\{\infty, \infty\}$. But, in any case, each fibre over a face of $\mathcal{Q}$ induced by the regular cover $\mathcal{R} \rightarrow \mathcal{Q}$ must have infinite cardinality.

Acknowledgements We want to thank Gabe Cunningham for insightful comments and the anonymous referees for valuable recommendations that have improved the paper. We are also particularly grateful to the Instituto de Matemáticas de la UNAM and allied sponsors for hosting the Second Workshop on Abstract Polytopes, Cuernavaca, Mexico, July 30-August 3, 2012.

\section{References}

1. Coxeter, H., Moser, W.: Generators and Relations for Discrete Groups, 4th edn. Springer, Berlin (1980)

2. Cunningham, G.: Mixing regular convex polytopes. Discrete Math. 312, 763-771 (2012)

3. Hartley, M.I.: All polytopes are quotients, and isomorphic polytopes are quotients by conjugate subgroups. Discrete Comput. Geom. 21, 289-298 (1999)

4. McMullen, P., Schulte, E.: Abstract Regular Polytopes. Encyclopedia of Mathematics and Its Applications, vol. 92. Cambridge University Press, Cambridge (2002)

5. Monson, B., Pellicer, D., Williams, G.: Mixing and monodromy of abstract polytopes. Trans. Anc. Monum. Soc. (in press)

6. Pellicer, D.: Extensions of regular polytopes with preassigned Schläfli symbol. J. Comb. Theory, Ser. A 116, 303-313 (2009)

7. Pellicer, D.: Developments and open problems on chiral polytopes. Ars Mat. Contemp. 5, 333-354 (2012)

8. Schulte, E.: On arranging regular incidence-complexes as faces of higher-dimensional ones. Eur. J. Comb. 4, 375-384 (1983)

9. The GAP Group: GAP-Groups, Algorithms, and Programming, Version 4.4 (2011). http://www. gap-system.org

10. Wilson, S.E.: Parallel products in groups and maps. J. Algebra 167, 539-546 (1994) 